\title{
RHBDF2 gene functions are correlated to facilitated renal clear cell carcinoma progression
}

Lei Wang, Xiu-Xiu Liu, Yu-Meng Yang, Yan Wang, Yuan-Yuan Song, Shan Gao, Lu-Yuan Li* and Zhi-Song Zhang* ${ }^{*}$

\begin{abstract}
Background: The rhomboids are a family of multi-transmembrane proteins, many of which have been implicated in facilitating tumor progression. Little is yet known, however, about rhomboid-associated biomarkers in cancers. An analysis of such biomarkers could yield important insights into the role of the rhomboids in cancer pathology.
\end{abstract}

Methods: In this study, we carried out the univariate Cox regression analysis and compared gene expression patterns of several rhomboid genes in 30 types of cancers by using The Cancer Genome Atlas (TCGA) database and the methods delineated in Gene Expression Profiling Interactive Analysis (GEPIA). We then used datasets GSE47032, GSE126964, GSE68417 and 75 paired pathological specimens to verify the influences of the rhomboid genes in cancer progression. Moreover, we carried out Weighted Gene Correlation Network Analysis (WGCNA) to investigate gene-related functions and we exploited potential correlations between rhomboid genes expression and immune cell infiltration in cancer tissues. Furthermore, we constructed gene-knockdown cancer cell lines to investigate rhomboid gene functions.

Results: We find that kidney renal clear cell carcinoma (KIRC) disease progression is affected by fluctuations in the expression of a number of the rhomboid family of genes and, more specifically, high levels of RHBDF2 gene expression are a good indicator of poor prognosis of the disease, as patients with high RHBDF2 expression levels exhibit less favorable survival rates compared to those with low RHBDF2 levels. Silencing of the RHBDF2 gene in KIRC cell lines leads to significantly diminished cell proliferation and migration; this is in good agreement with the identification of an enhanced presence of a number of cell growth and migration promoting signaling molecules in KIRC tumors. We found that, although high level of RHBDF2 correlated with increased infiltration of lymphocytes in cancer tissues, artificially overexpressed RHBDF2 led to an inhibition of the activity of the infiltrated immune cells through sustaining PD-L1 protein level. Furthermore, we show that RHBDF2 related cell migration and PD-L1 regulation were potentially mediated by EGFR signaling pathway.

Conclusions: RHBDF2 gene functions are correlated to facilitated renal clear cell carcinoma progression and may serve as a critical prognostic biomarker for the disease.

Keywords: Kidney renal clear cell carcinoma, Rhomboids, RHBDF2, Cancer prognosis, PD-L1

*Correspondence: liluyuan@nankai.edu.cn; zzs@nankai.edu.cn State Key Laboratory of Medicinal Chemical Biology, College of Pharmacy, Tianjin Key Laboratory of Molecular Drug Research, Nankai University, 38 Tongyan Road, Jinnan District, Tianjin 300350, China

\section{Background}

Members of the rhomboid superfamily are six- or seventransmembrane proteins, which have been shown to be widely present and may take part in a variety of original author(s) and the source, provide a link to the Creative Commons licence, and indicate if changes were made. The images or other third party material in this article are included in the article's Creative Commons licence, unless indicated otherwise in a credit line to the material. If material is not included in the article's Creative Commons licence and your intended use is not permitted by statutory regulation or exceeds the permitted use, you will need to obtain permission directly from the copyright holder. To view a copy of this licence, visit http://creativecommons.org/licenses/by/4.0/. The Creative Commons Public Domain Dedication waiver (http://creativeco mmons.org/publicdomain/zero/1.0/) applies to the data made available in this article, unless otherwise stated in a credit line to the data. 
important biological processes, including cytokine secretion [1-5], protein quality control [6-9], epithelial cell polarity [10], subcellular transport [11], and mitochondrial function regulation [12]. They are also shown to be associated with disease development such as in cancers [13-17] and autoimmune diseases [18-20]. Rhomboid proteins may be categorized in two groups, namely the proteolytically active or inactive [21]. The proteolytically active rhomboids include RHBDD1, RHBDL1, RHBDL2, RHBDL3 and PARL [22], that are capable of catalyzing the cleavage of their substrates and regulating related pathways. For example, they cleavage the pro-ligands of epidermal growth factor receptor (EGFR) and activate the EGFR signaling pathway $[4,16,23]$. The proteolytically inactive group includes RHBDF1, RHBDF2 (also known as iRhom1 and iRhom2, respectively) [24] were also able to activate the EGFR pathway through activation of EGFR ligands. RHBDF1 participates in GPCRmediated transactivation of EGFR growth signals and RHBDF2 drives EGFR activation through an enhancement of the secretion of EGFR ligands [5, 13, 14]. The role of rhomboids in cancer progression is an important but under-explored subject in cancer research. We therefore set out to explore the gene expression patterns of rhomboids in a variety of cancers and their relationships to cancer progression, with a specific attention to associated biomarkers.

Kidney cancers account for approximately $2 \%$ of adult malignancies and is among the most prevalent cancers worldwide [25]. About $80 \%$ of kidney cancer cases are renal clear cell carcinoma (KIRC) [26]. In addition, symptoms of KIRC are often insidious in the early stage, which could explain why many patients are diagnosed when the disease is already in the advanced stages [27, 28]. The five-year overall survival (OS) rate of KIRC patients at early stage is up to $90 \%$, whereas the disease becomes almost incurable in advanced stages[29]. Chemotherapy or partial resection are the main methods of treatment, yet local recurrence or distant metastasis often occurs [30]. In recent years, immune checkpoint inhibitors (ICI) which can block the PD-1/PD-L1 or CTLA-4 T cell inhibitory receptor, have been shown to be encouragingly effective in advanced renal-cell carcinoma [31, 32]. But some effects of immunotherapy are not durable. Discovery of new biomarker for disease progression or treatment option will undoubtedly facilitate the diagnosis and treatment of the disease [33].

In this study we determined the gene expression patterns of rhomboids in 30 types of cancers by using The Cancer Genome Atlas (TCGA) and the Gene Expression Profiling Interactive Analysis (GEPIA) databases.
We then focused on RHBDF2 expression in KIRC and analyzed lymphocyte infiltration, immune checkpoints, and their relationships to disease progression. Our findings indicate that $R H B D F 2$ gene functions significantly contribute to the immunosuppressive microenvironment in KIRC. These data are consistent with the view that increased RHBDF2 may serve as a critical biomarker of poor prognosis of renal clear cell carcinoma as well as a potential therapeutic target.

\section{Methods}

\section{Data source}

Gene expression data of 30 types of cancers in TCGA database (https://portal.gdc.cancer.gov/) was downloaded from GDC API, and all sequencing data was normalized to TPM. Essential pathologic information and survival data of each individual cancer case were from TCGA database and TCGA Pan-Cancer Clinical Data Resource [34]. Gene expression datasets, GSE68417, GSE126964, GSE47032 and GSE167093, were downloaded from Gene Expression Omnibus (GEO) databases (https://www.ncbi.nlm.nih.gov/gds/). The GSE68417 [35] series on the platform GPL6422 (Affymetrix Human Gene 1.0 ST Array) includes 14 normal samples, 6 tumor benign samples, 13 low grade samples and 16 high grade samples of KIRC. The GSE126964 [36] series on the platform GPL20795 (HiSeq X Ten) contains 55 tumor samples and 11 matched normal samples from Chinese KIRC patients. The GSE47032 [37] series on the platform GPL5175 (Affymetrix Human Exon 1.0 ST Array) contains 10 KIRC tumor samples and their matched non-tumor samples. The GSE167093 on the platform GPL10558 (Illumina HumanHT-12 V4.0 expression beadchip) contains 609 renal tumors and 256 non-tumor renal tissues [38].

\section{Gene expression analysis in GEPIA}

Rhomboid genes expression difference between tumor and normal tissues of adrenocortical carcinoma (ACC), KIRC and brain lower grade glioma (LGG) was analyzed by GEPIA [39] with the datasets in TCGA and The Genotype-Tissue Expression projects (GTEx). GEPIA, a web-based tool, (http://gepia.cancer-pku.cn/index.html) provides multiple interactive functions including differential expression analysis.

\section{Immune infiltration related analysis}

We first analyzed the infiltration of immune cells in KIRC and GSE68417 with the ESTIMATE algorithm [40] (https://bioinformatics.mdanderson.org/estimate/rpack 
age.html). Then we calculated the Pearson correlation coefficient of immune infiltration scores and RHBDF2 expression and drew the scatter plot with "ggstatsplot" $\mathrm{R}$ package (https://cran.r-project.org/web/packages/ggsta tsplot/index.html). The infiltrating immune cell types analysis was performed by TIMER2.0 (http://timer.cistr ome.org/) which is an online analysis tool for scoring immune infiltrates across diverse cancer types by multiple immune deconvolution methods, including TIMER, CIBERSORT, quanTIseq, xCell, MCP-counter and EPIC algorithm [41-43]. Gene expression data in KIRC-TCGA was analyzed by TIMER algorithm, and patients were grouped according to the median of $\mathrm{CD} 8^{+} \mathrm{T}$ cell infiltration scores or macrophage infiltration scores for further survival analysis. Survival curve drawing was completed by $\mathrm{R}$ package, "survminer" (https://cran.r-project.org/ web/packages/survminer/index.html).

\section{Functional enrichment analysis}

Weighted Gene Correlation Network Analysis (WGCNA) was performed with R package "WGCNA" (https://cran.r-project.org/web/packages/WGCNA/ index.html) to identify significant functional modules based on RHBDF2 expression in KIRC. The appropriate soft threshold and co-expression modules were obtained first, and then we acquired the Pearson correlation coefficient between each module and RHBDF2 expression. Three modules related to RHBDF2 expression were determined at last. The annotation of gene functions and gene-interactive networks in the RHBDF2-related modules were carried out by Network Analyst [44-48] (https://www.networkanalyst.ca/) and GeneMANIA [49] (http://genemania.org). The functional enriched pathways, depicted in Sankey diagram, was completed by $R$ package "ggalluvial" [50]. Gene Set Enrichment Analysis (GSEA) was also performed by grouping the TCGA-KIRC with the median of RHBDF2, which was accomplished through locally downloaded GSEA software (https://www.gsea-msigdb.org) [51, 52].

\section{Materials}

The human KIRC cell lines, 786-O and 769-P, were purchased from Cell Bank of the Chinese Academy of Sciences (Shanghai, China). The cells were cultured in RPMI1640 medium supplemented with 10\% FBS. The pathological specimens of human renal clear cell carcinoma, including 75 paired tumor and adjacent tissues, were purchased from Shanghai Outdo Biotech Co., Ltd. RHBDF2 antibody (Proteintech, \#23181-1-AP) was used for immunohistochemical staining and Western blotting.
PD-L1 antibody (Boster, \#BA1683-2), EGFR antibody (BBI, \#D160292), phospho-EGFR antibody (Boster, \#BM4676) were used for Western blotting. BeyoClick ${ }^{\mathrm{TM}}$ EdU Cell Proliferation Kit with Alexa Fluor 488 (Beyotime, C0071S) was used to detect cell proliferation according to the method described in the product manual. Cell total RNA extraction was accomplished by EasrepTM total RNA extraction kit (Progmega, LS1040) and Hifair ${ }^{\circledR}$ III 1st Strand cDNA Synthesis SuperMix (Yeasen, 11120 ES60) was used for reverse transcription. UltraSYBR mixture (CWBIO, CW0957M) was use to real-time quantitative PCR. Matrigel (Corning \#354248) was used to build the subcutaneous graft model. Gefitinib (Topscience \#T1181) was used to block EGFR activation.

\section{Real-time quantitative PCR (RT-qPCR)}

Hub genes of the WGCNA red module and immune checkpoints were detected by RT-qPCR. Total RNA extraction from the 786-O and 769-P cells were accomplished according to the manual of RNA extraction kit. Then we took $1 \mu \mathrm{g}$ RNA for cDNA synthesized and targeted genes detection. The thermal cycle program was as follows: denaturing for $15 \mathrm{~s}$ at $95{ }^{\circ} \mathrm{C}$, annealing and extension for $30 \mathrm{~s}$ at $60{ }^{\circ} \mathrm{C}$. Relative expression of the targeted genes was normalized to the ACTB by calculating the delta-Ct. Primers for RT-qPCR were as follows:

ITGB1-F: CAAGAGAGCTGAAGACTATCCCA,

ITGB1-R: TGAAGTCCGAAGTAATCCTCCT, MAPK3-F: ATGTCATCGGCATCCGAGAC, MAPK3-R: GGATCTGGTAGAGGAAGTAGCA, PTK2-F: TGGTGCAATGGAGCGAGTATT, PTK2-R: CAGTGAACCTCCTCTGACCG, CD273-F: ACCGTGAAAGAGCCACTTTG CD273-R: GCGACCCCATAGATGATTATGC CD276-F: GTCCCTGAGTCCCAGAGTCG CD276-R: ACGCAGCATCTTCCTGTGAG LGALS9-F: TCTGGGACTATTCAAGGAGGTC LGALS9-R: CCATCTTCAAACCGAGGGTTG ACTB-F: CATGTACGTTGCTATCCAGGC, ACTB-R: CTCCTTAATGTCACGCACGAT,

\section{RHBDF2 knocked-down cell lines construction}

Two independent shRNAs against RHBDF2 mRNA were synthesized by Genweiz, the sequences of which were (forward):

\#1: GTGAAGCACTTTGCCTTTGATCTCGAGATC AAAGGCAAAGTGCTTCAC

\#2: CACGGCTATTTCCATGAGGAACTCGAGTTC CTCATGGAAATAGCCGTG. 
RHBDF2-shRNA sequences were constructed into pLKO.1 plasmid, and then transfected into $293 \mathrm{~T}$ cells at a ratio of pLKO.1: psPAX2: pMD2.G = 4:2:1. The supernatant of culture medium was collected after 48 hours and filtered by a $0.45 \mu \mathrm{m}$ filter to obtain the virus crude solution. The virus solution was used to infect 786-O cells and 769-P cells with the addition of $10 \mu \mathrm{g} / \mathrm{mL}$ of Polybrene. The control group of 786-O and 769-P were infected with empty vector virus. Cell culture media was changed after 24 hours, and puromycin was used to screen the infected cells with a $2 \mu \mathrm{g} / \mathrm{mL}$ concentration. Three days later, 786-O cells and 769-P cells with RHBDF2 knocked-down were obtained.

\section{Scratch healing assay}

$1 \times 10^{5}$ tumor cells were seeded in 12 -well plate and cultured with $10 \%$ FBS-RPMI1640 medium under $37^{\circ} \mathrm{C}$ and $5 \% \mathrm{CO}_{2}$ overnight. Then we draw a straight line in the middle of each well to create the wound and washed every well twice with PBS and change the medium to $2 \%$ FBS-RPMI1640. The microscopic images were taken by Nikon ECLIPSE Ts2 after 12 and 24 hours.

\section{Trans-well assay}

$3.5 \times 10^{4}$ cells were seeded into the upper chamber suspending in 2\% FBS RPMI1640 medium, while in the lower chamber the concentration of FBS was $20 \%$. After cultured for 24 hours, cells were fixed with $4 \%$ paraformaldehyde for 15 mins at room temperature. Cells at the upper side were then erased and those at the bottom side were stained by $0.1 \%$ crystal violet solution. After washed with PBS, the bottom of the chamber was photographed and analyzed.

\section{Mouse model}

We used 786-O cells and five-week-old nude mice to build the subcutaneous xenograft model. 786-O cells infected with empty virus or RHBDF2-shRNA virus were cultured in vitro and seeded into the abdominal subcutaneous tissue of mice. We mixed Matrigel and RPMI1640 medium at a 1:1 ratio, and then used the mixed medium to inject $1 \times 10^{7}$ cells per mouse.

\section{Statistical analysis}

For the computerized analyses, we used the data of TCGA to carry out the univariate Cox regression and survival analyses, and the log-rank test was used for the significance test. WGCNA and GSEA were performed for RHBDF2 related functions and signaling pathways. Correlations between RHBDF2 expression and other factors mentioned in the study were mainly determined by the Pearson correlation coefficient. All the experiments in vitro in the study have been repeated at least 3 times. Data were subjected to student t-test, one-way ANOVA or two-way ANOVA for the statistical significance analyses. $P$-values of the differences smaller than 0.05 were of significance. For detail, the statistical analyses of each experiment were depicted in the relevant figure legends.

\section{Results}

Assessment of the prognostic value of rhomboid proteins in cancers

In order to begin to explore potential correlation between rhomboid functions and cancers, we carried out the univariate Cox regression analysis of the rhomboids family of genes in 30 cancers from TCGA database. The value of Hazard Ratio (HR) was used to describe the influence of the gene expression on tumors (Fig. 1). We found that the expression of rhomboids was most significantly correlated with three types of cancers, namely ACC, LGG and KIRC. The results of HR (Fig. 2); (more details are given in Additional file 1: Table S1) and gene expression patterns analyzed by GEPIA (Additional file 2: Fig.S1) demonstrated that four genes, RHBDD3, RHBDL2, RHBDF1 and RHBDF2, exhibited prognostic value in KIRC. Additionally, high levels of RHBDD1 and RHBDF2 are correlated to poor prognosis of LGG, whereas RHBDD2 and PARL are markedly correlated to ACC disease progression. More importantly, most members of the rhomboid family displayed negative effect on KIRC prognosis. It is worthwhile to point out that RHBDF2 expression is significantly up-regulated in $\mathrm{KIRC}$, in comparison to other rhomboids, suggesting a potential prognostic value.

\section{Validation the prognostic role of RHBDF2 in KIRC}

We then focused on the prognostic value of RHBDF2 for KIRC (Fig. 3a-c). We found that patients in RHBDF2high group exhibited markedly shorter overall survival (OS), disease-specific survival (DSS), and progressionfree survival (PFS) rate. Additionally, we analyzed the relationship between RHBDF2 and clinical parameters recorded in TCGA database. The diagnosed ages and $T, N, M$ stages showed two distinctive differences in RHBDF2-high and low groups. Larger proportion of patients younger than 60-year-old or patients with advanced cancers had higher expression level of RHBDF2 (Table 1). Meanwhile, RHBDF2 had an increased expression in tumor tissue, which was validated in datasets GSE47032, GSE68417, and GSE126964 (Fig. 3d-f) and 


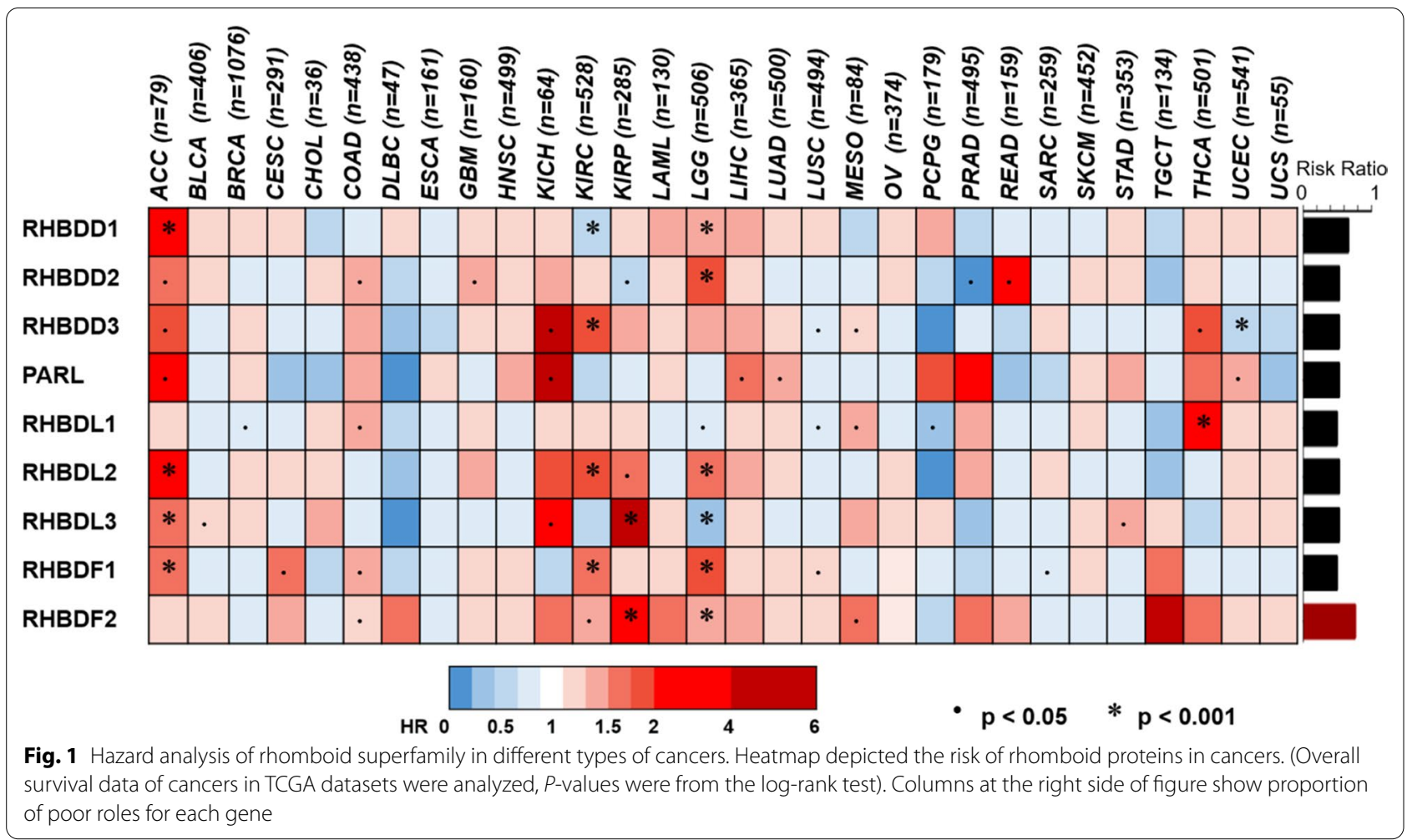

that high RHBDF2 levels were positively correlated with tumor grades (Fig. 3g, h). We performed immunohistochemical staining in the specimens of KIRC patients with different stages and tumor grades, and found that the RHBDF2 protein was substantially more abundant in high-grade tumors (Fig. 4).

\section{RHBDF2 overexpression in KIRC correlates} with an immunosuppressive microenvironment

Since KIRC has a character of high lymphocytes infiltration (31), we analyzed the immune infiltration levels in the KIRC samples with gene expression data in TCGA database and GSE68417 using R package "ESTIMATE". And the results of correlation analysis showed that RHBDF2 was positively correlated with enhanced immune infiltration (Fig. 5a, b). We then divided the KIRC samples into immune infiltration score-high (IMS-high) and -low (IMS-low) groups, and carried out survival analysis. We found that KIRC patients in the IMS-high group exhibited a shorter survival period compared to those in the IMS-low group (Fig. 5c). We further divided the KIRC samples into four groups: immune infiltration-high and
RHBDF2 expression-high (IMS-high/R2-high), IMShigh/R2-low, IMS-low/R2-high and IMS-low/R2-low. Disease specific survival analysis of these specimens indicated that patients in IMS-high/R2-high group had the shortest survival time (Fig. 5d).

It was reported previously that KIRC patients with high macrophage and $\mathrm{CD} 8^{+} \mathrm{T}$ cell infiltration survived poorly [53]. We scored the infiltrated lymphocytes by TIMER 2.0 with data in KIRC-TCGA and conducted survival analyses based on immune infiltration scores and RHBDF2 expression levels. We found that patients with both high level of macrophages infiltration and RHBDF2 expression suffered shorter survival time than those in other groups (Fig. 5e). Additionally, patients with higher $\mathrm{CD}^{+} \mathrm{T}$ cell infiltration and lower RHBDF2 expression exhibited favorable prognosis, whereas patients in higher $\mathrm{CD} 8^{+} \mathrm{T}$ cell infiltration and higher RHBDF2 expression group exhibited the poorest prognosis (Fig. 5f). These findings suggest that high levels of RHBDF2 prominently affect the anticancer activities of macrophages and $\mathrm{CD} 8^{+} \mathrm{T}$ cells. 


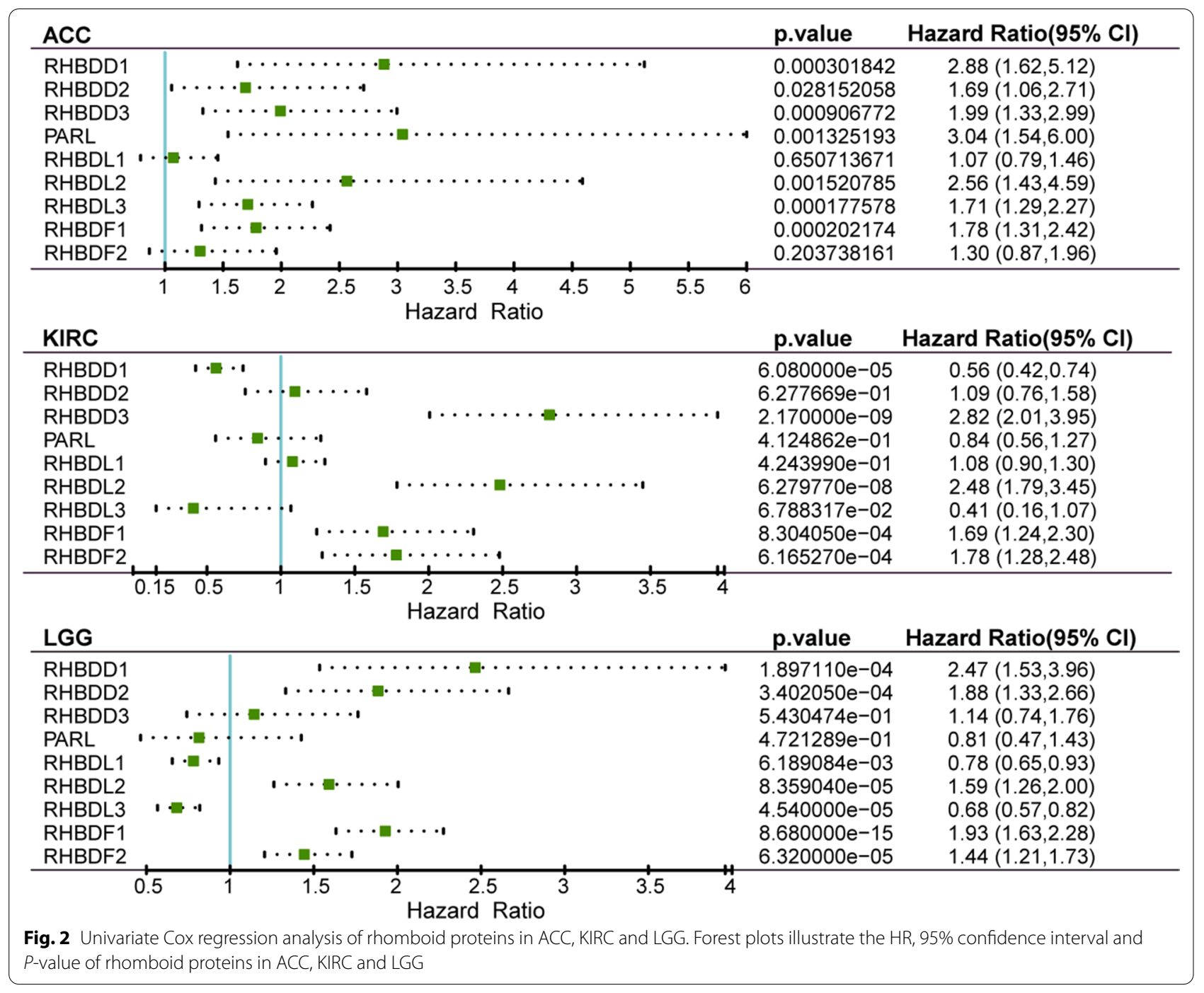

\section{RHBDF2 gene-silencing leads to reduction of PD-L1 level in renal cancer cells}

Overexpressed RHBDF2 inhibited the function of infiltrated immune cells, which suggests us to analyze the expression of immune checkpoints molecules. We evaluated immunosuppressive checkpoints expression in tumor tissues and normal tissue. CD274, CD273, VTCN1, CD276, LGALS9 and CMTM4 were up-regulated in KIRC tissues (Additional file 3: Fig. S2). Then we analyzed the Pearson correlation between those checkpoints and RHBDF2 in TCGA and GEO datasets. The expression of CD273, CD276 and LGALS9 had stronger positive correlation with $R H B D F 2$. The correlation of $R H B D F 2$ between $C D 274$ and CMTM6 were also light. However, VTCN1 and CMTM4 were negatively correlated to RHBDF2 (Additional file 4: Fig. S3a). Positive correlation of $C D 273$ and $C D 276$ with the expression of $R H B D F 2$ was detected in 786-O and 769-P cells by RTqPCR (Additional file 4: Fig. S3b).

PD-L1, the product of the CD274 gene, is an immune checkpoint protein highly expressed in renal carcinoma [54]. We measured the transcription relevance between RHBDF2 and CD274 in the databases but found no significant correlations (Fig. 6a-d). We then prepared shRNA lentivirus to artificially knockdown RHBDF2 in renal cancer cell-lines (786-O and 769-P). We found that protein level of PD-L1 decreased in RHBDF2 knockeddown cells (Fig. 6e-h). These findings suggested that RHBDF2 may have a potentially important role in the 
a.

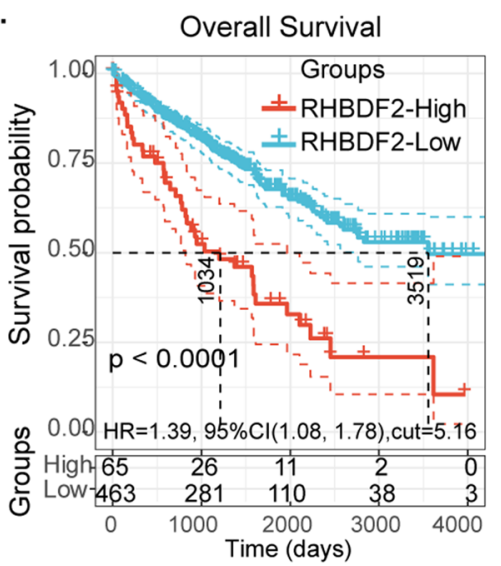

d.

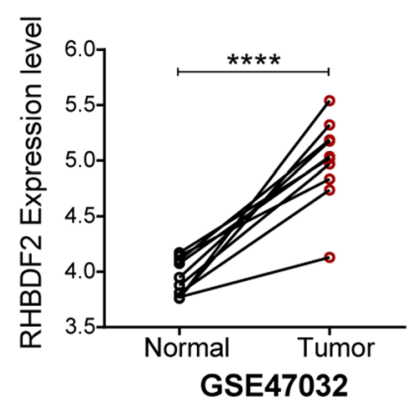

b.

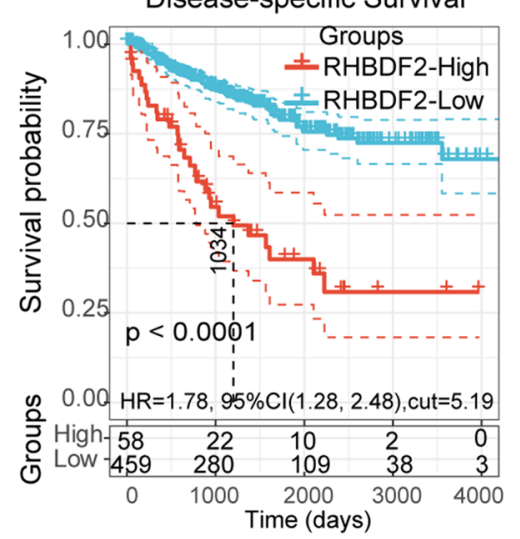

e.

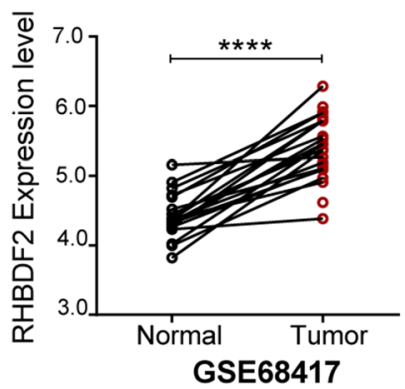

C.

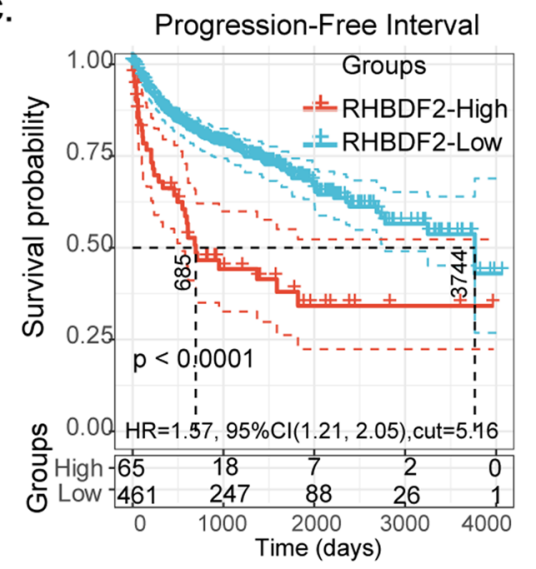

f.

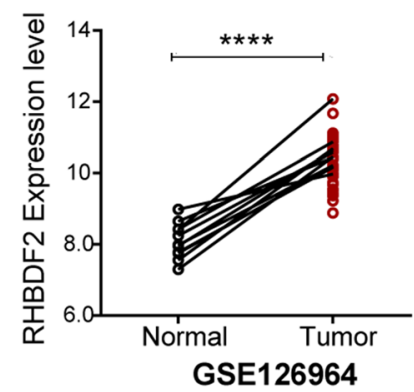

g.
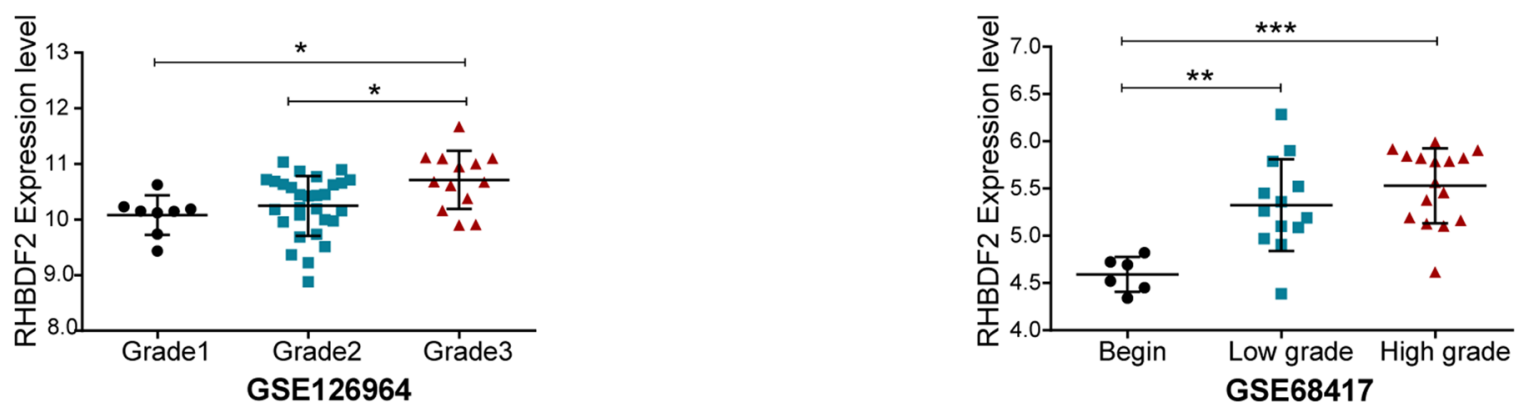

Fig. 3 Association between RHBDF2 expression and tumor malignancy in KIRC. Survival analysis of RHBDF2 in KIRC was performed with data in TCGA database. a Overall survival analysis. b Disease-specific survival analysis. c Progression-free interval analysis were present between RHBDF2-high and -low expression groups ( $P$-value was from the log-rank test). $\mathbf{d}-\mathbf{f}$ RHBDF2 expression between normal and tumor tissues in datasets GSE47032, GSE68417 and GSE126964 respectively (GSE47032, $\mathrm{n}=20 ; \mathrm{GSE1}$ 26964, $\mathrm{n}=66 ; \mathrm{GSE68417,} \mathrm{n}=49 ; P$-value was from the paired t-test). g, h RHBDF2 expression with different tumor grades in GSE126964 and GSE68417 ( $P$-value was from one-way ANOVA; ${ }^{*} p<0.05,{ }^{* *} p<0.01$, $\left.{ }^{* * *} p<0.005,{ }^{* * *} p<0.001\right)$ 
Table 1 Clinical characteristics of patients according to RHBDF2 expression in KIRC-TCGA

\begin{tabular}{|c|c|c|c|c|}
\hline \multirow[t]{2}{*}{ Characteristics } & \multirow[t]{2}{*}{ Total number } & \multicolumn{2}{|c|}{$\begin{array}{l}\text { RHBDF2 Expression in } \\
\text { Groups }\end{array}$} & \multirow[t]{2}{*}{$P$-value } \\
\hline & & Low (\%) & High (\%) & \\
\hline \multicolumn{5}{|l|}{ Laterality } \\
\hline Left & 249 & $117(44.15 \%)$ & $132(50.00 \%)$ & \multirow[t]{2}{*}{ ns } \\
\hline Right & 280 & $148(55.85 \%)$ & $132(50.00 \%)$ & \\
\hline \multicolumn{5}{|l|}{ Age } \\
\hline$\leq 60$ & 261 & $113(42.64 \%)$ & $148(55.85 \%)$ & \multirow[t]{2}{*}{$<0.01$} \\
\hline$>60$ & 269 & $152(57.36 \%)$ & $117(44.19 \%)$ & \\
\hline \multicolumn{5}{|l|}{ Pathologic T } \\
\hline $\mathrm{T} 1-2$ & 350 & $186(67.64 \%)$ & 164 (61.89\%) & \multirow[t]{2}{*}{$<0.001$} \\
\hline T3-4 & 190 & 89 (32.36\%) & $101(38.11 \%)$ & \\
\hline \multicolumn{5}{|l|}{ Pathologic N } \\
\hline Mo & 420 & 225 (87.89\%) & 195 (80.58\%) & \multirow[t]{2}{*}{$<0.02$} \\
\hline $\mathrm{M} 1$ & 78 & $31(12.11 \%)$ & 47 (19.42\%) & \\
\hline \multicolumn{5}{|c|}{ Pathologic stage } \\
\hline Stage I & 265 & $143(54.17 \%)$ & $122(46.39 \%)$ & \multirow[t]{2}{*}{$<0.05$} \\
\hline Stage II-IV & 265 & 121 (45.83\%) & 141 (53.61\%) & \\
\hline
\end{tabular}

$P$-value was from a Chi-square test

maintenance of immune checkpoints level in renal cancer cells, which could make RHBDF2 a valuable target in assisting immunotherapy.

\section{Key modules identification and functional annotation analysis}

In order to further investigate the biological characteristics of renal clear cell carcinoma that are associated with various levels of RHBDF2 expression, we carried out WGCNA. Messenger RNA profiles of the specimens with similar patterns were grouped into several functional modules based on WGCNA calculation. These operations revealed a total of 53 modules (Additional file 5: Fig. S4a). The transcription level of RHBDF2 was used as a characteristic trait for grouping. We determined the relevance between each functional module and grouping trait by Pearson correlation analysis, and identified the top three modules correlated with RHBDF2 level and marked them, respectively, in green, red and orange (Fig. 7a, Additional file 5: Fig. S4b-c, Additional file 6: Table S2). We determined the hub genes in the green, red and orange modules by using Network Analyst (Additional file 7: Table S3). The hub genes in each module were used to carry out the gene-interactive network analysis and annotate the module-related functions by GeneMANIA. The results of gene network analyses were shown in Additional file 8: Fig. S5, Additional file 9: Fig. S6, Additional file 10: Fig. S7. Functional annotation of hub module genes was shown in a bubble diagram (Additional file 11: Fig. S8). The function of orange module genes was related to the cell morphology and cell cytoskeleton, the function of green module genes was related to mRNA processing, membrane fusion and cell cycle, and the genes in red module mostly show connections with cell junction, cell migration and growth factor receptor signaling pathways (Fig. $7 \mathrm{~b}$ ).

\section{RHBDF2 gene-silencing restricts renal clear cell cancer cell proliferation and migration}

To verify the results from bioinformatics analysis that the genes and pathways related to RHBDF2 functions may contribute to cancer cell migration and signal transduction, we silenced the $R H B D F 2$ gene by using shRNA in renal clear cell carcinoma cell line 786-O and 769-P. The cell-cycle was measured by Edu-incorporation in which all cell nuclei were stained blue with Hoechst33342 and the nuclei of cells in S-phase were labeled with green fluorescence. We found that the proportion of green fluorescence decreased in $R H B D F 2$ gene knocked-down cells, indicating a decreased proliferation rate (Fig. $8 \mathrm{a}, \mathrm{b}$ ). The decrease of cell proliferation rate as a result of RHBDF2 knockdown was verified by MTT assay (Fig. 8c). Moreover, we carried out trans-well and scratch healing assays, and found that RHBDF2 knockdown gave rise to significantly reduced cell motility in $786-\mathrm{O}$ cells and 769-P cells in comparison with the scramble shRNA treated cells (Fig. 8d-g). Furthermore, we used quantitative real-time PCR to detect migration related hub genes obtained by enrichment analysis, and found that the transcription of ITGB1, MAPK3 and PTK2 were significantly decreased in RHBDF2 knocked-down cells (Fig. 8h). 


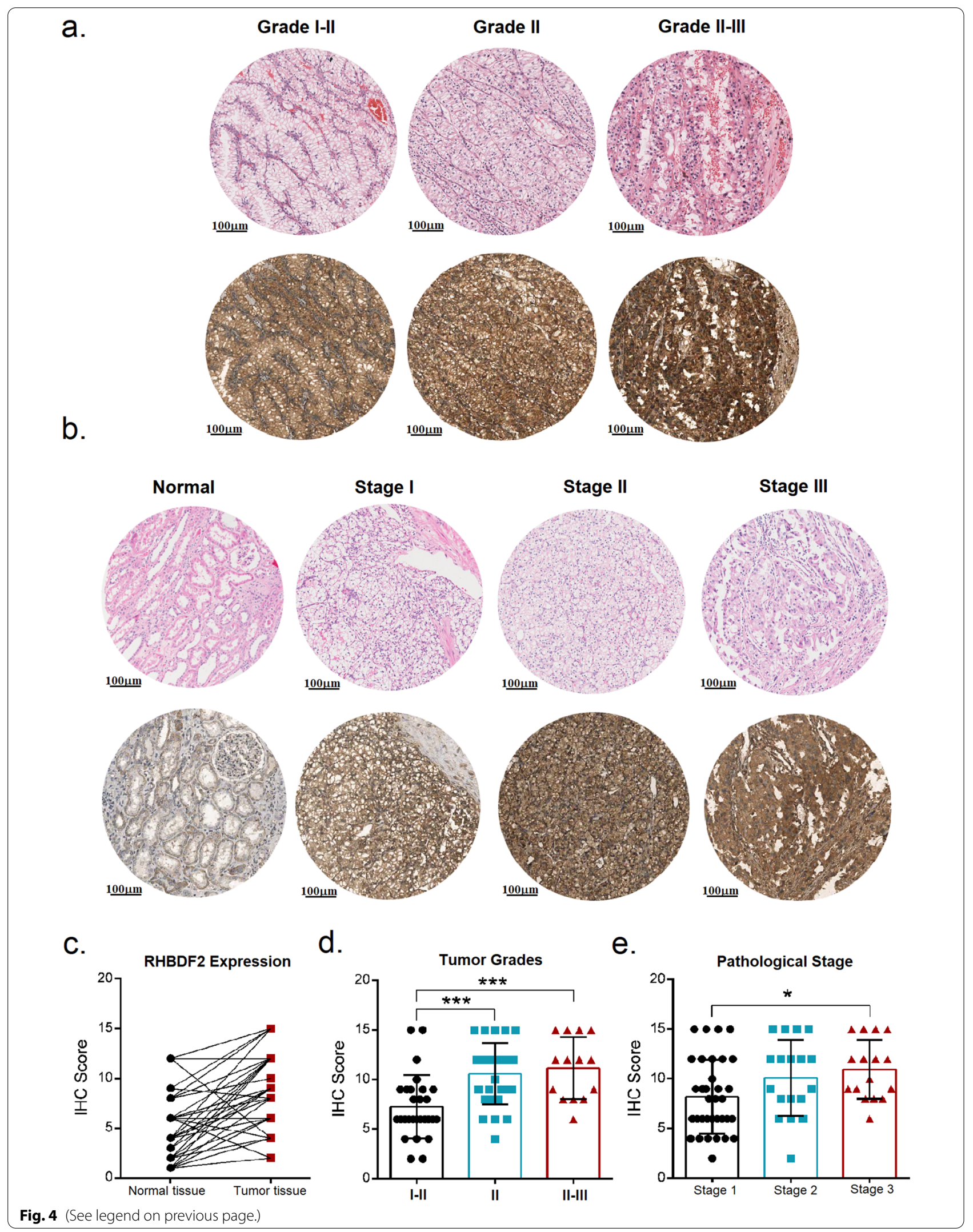


To investigate the function of RHBDF2 in vivo, we constructed a xenograft model based on the KIRC cell line 786-O in nude mice. As shown in Fig. 9c, d, the tumor growth rate in the RHBDF2-knockdown group was significantly slower than that in the control group. At the same time, we also observed a delay in the early stage of tumor formation in RHBDF2-knockdown group.

\section{RHBDF2 related functions were mediated by EGFR signaling pathway}

We then focus on the signaling that RHBDF2 mediated. We used the data of KIRC-TCGA to carry out the GSEA, combined the results with WGCNA and obtained the functional enrichment results of RHBDF2 in both analysis (Additional file 12: Table S4). We found the enrichment results of the red module in WGCNA were more similar to those of GSEA (Additional file 13: Fig. S9). Also, as shown in Fig. 7b, we presented the pathways that RHBDF2 possibly regulated, the enriched ones of which contained EGFR, ERBB and FGFR. In Fig. S5, the hub genes of the red module, like PTK2, MAPK3, were also connected to the EGFR pathway, as a role of the downstream of signaling or interaction, which suggested the correlation of RHBDF2 and EGFR. Meanwhile, we detected the reduction of phosphoEGFR in RHBDF2 knockdown cells (Fig. 9a, b), and the phenomenon was also remarkable in the tumor sections of 786-O xenografts (Fig. 9e, f). We used EGFR inhibitor Gefitinib to significantly reduce the migration ability of 786-O and 769-P cells (Fig. 9g, h), which was consistent with the effect of RHBDF2-knockdown treatment. Based on the above results, we speculate that the EGFR signaling pathway may be the main mediator of RHBDF2's regulation on cell invasion and migration.

EGFR pathway was also reported to regulate PD-L1 level [55]. When we used Gefitinib to block EGFR signaling, the protein level of PD-L1 showed a reduction (Fig. 9i, j). In view of RHBDF2 knockdown significantly reduced EGFR activation, it's plausible that EGFR pathway was an important signaling pathway by which RHBDF2 regulates PD-L1.

\section{Discussion}

The primary goal of this study is to gain initial insights into the role of the rhomboid family of genes in cancer progression in general, and that of the inactive rhomboids more specifically. The challenge from this study is the need to integrate a large amount of prognostic information of 30 cancers and the expression abundance of rhomboids in different cancers. The approach leads us to a more holistic understanding of the role of rhomboids in cancer. Our findings indicate that KIRC disease progression is highly likely to be affected by fluctuations of rhomboids gene expressions and, more specifically, by high levels of RHBDF2 gene expression. And RHBDF2 is a good indicator of poor prognosis of the disease. Advanced analyses of KIRC, like WGCNA, GO/KEGG pathways annotation pointed to the regulatory role of RHBDF2 in cell proliferation and migration, implicating its potential as a target for cancer therapy.

The result of immune infiltration analysis is interesting, high levels of RHBDF2 positively correlates with infiltration of lymphocytes in cancer tissues but is not conductive to the survival of patients. By analyzing the immune suppressive checkpoints level, we confirmed that RHBDF2 show positive correlation with checkpoints, like CD273 and CD276. Moreover, we found that RHBDF2 functions may be critically required in establishing high PD-L1 protein levels in cancer cells, which suggests that RHBDF2 be a valuable therapeutic target in line with PD-L1-focused immunotherapy, in addition to being of diagnostic and prognostic factor for renal clear cell cancer. The question of enhanced immune infiltration into the tumors is particularly interesting. A previous report of KIRC tumors [56] identified three distinct groups, namely immune silencing, immune activation and immune regulation, based on the infiltration of $\mathrm{CD}^{+} \mathrm{T}$ cells. $\mathrm{T}$ cells in the immune-regulated group are

\footnotetext{
(See figure on next page.)

Fig. 5 Immune cell infiltration and RHBDF2 expression in survival analysis. Scatter diagram depicted the correlation between immune infiltration scores and RHBDF2 expression with a KIRC samples in TCGA database and b in GSE68417. c Survival analysis for patients with high or low immune infiltration. d Survival analysis grouping with immune infiltration scores and RHBDF2 expression. e Survival analysis based on the macrophage infiltration scores and RHBDF2 expression (MHRH: macrophage-high infiltration and RHBDF2-high expression; MHRL: macrophage-high infiltration and RHBDF2-low expression; MLRH: macrophage-low infiltration and RHBDF2-high expression; MLRL: macrophage-low infiltration and RHBDF2-low expression). f Disease specific survival analysis were present, which grouped with $\mathrm{CD}^{+} \mathrm{T}$ cell infiltration and $\mathrm{RHBDF} 2$ expression (THRH: CD8 ${ }^{+} \mathrm{T}$ cell-high infiltration and RHBDF2-high expression; THRL: CD8 ${ }^{+}$T cell-high infiltration and RHBDF2-low expression; TLRH: CD8 ${ }^{+} \mathrm{T}$ cell-low infiltration and RHBDF2-high expression; TLRL: CD8 ${ }^{+}$T cell-low infiltration and RHBDF2-low expression). P-values were from log-rank tests. Data in figure c-f were derived from TCGA
} 
a.

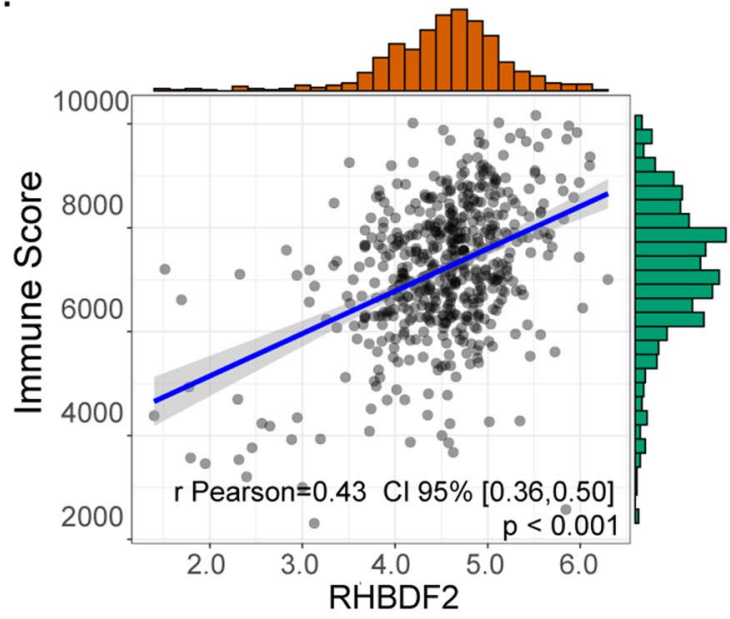

C.

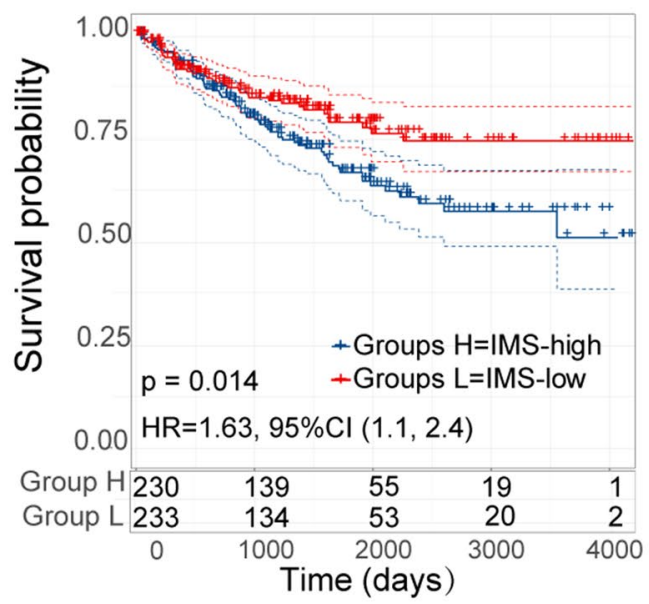

e.

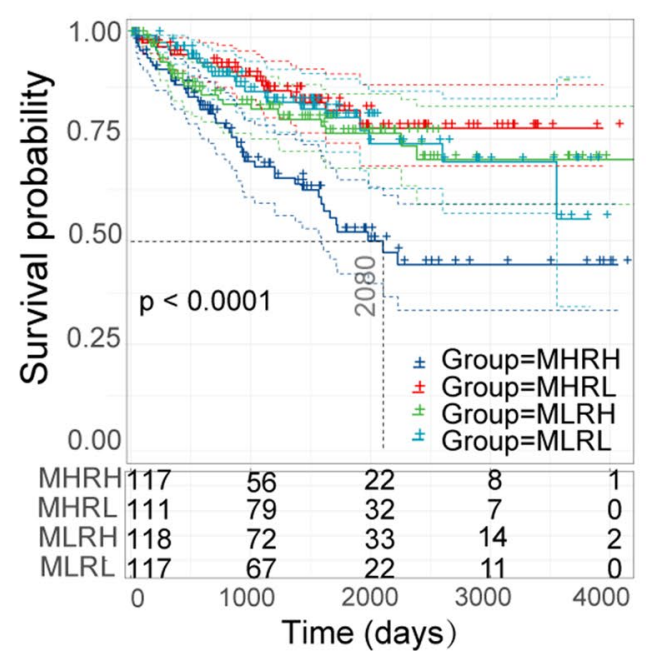

b.

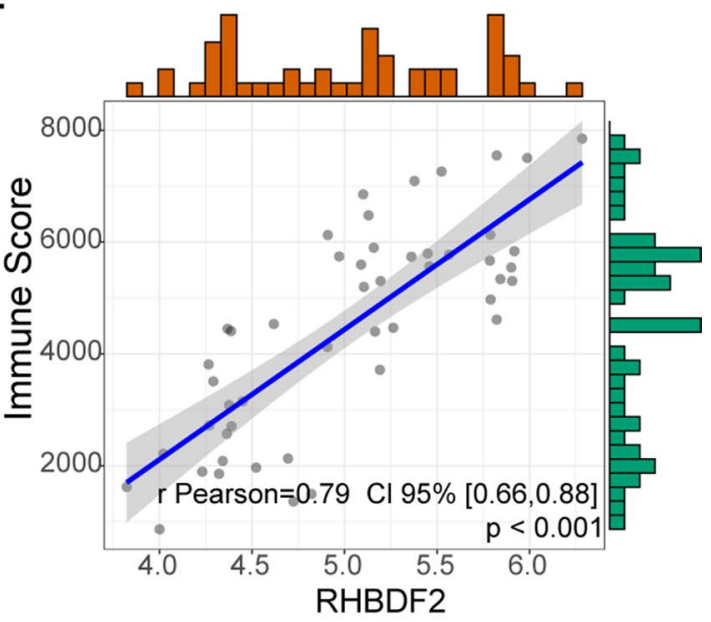

d.

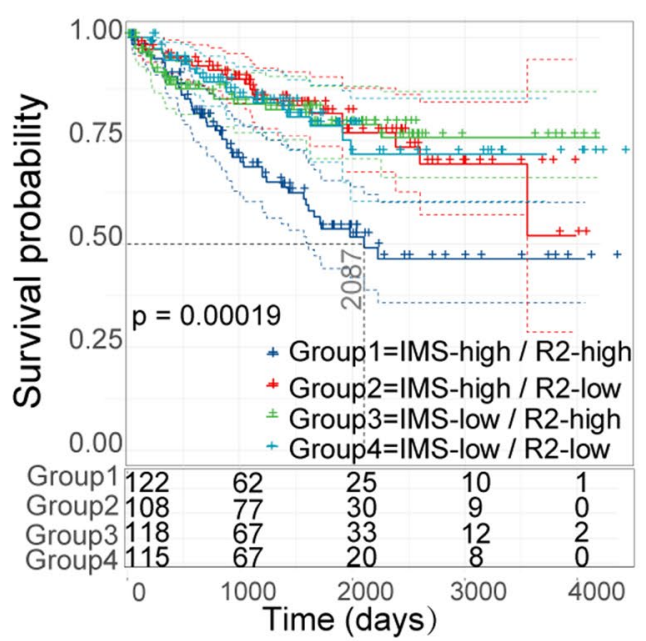

f.

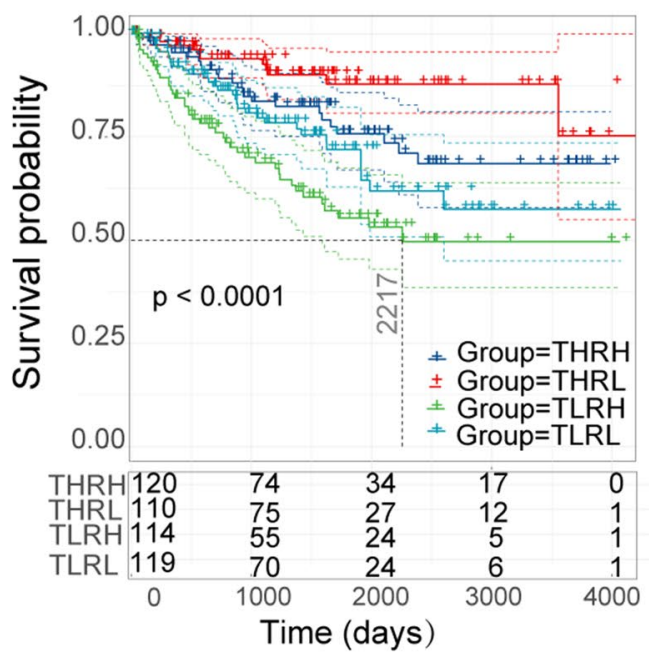

Fig. 5 (See legend on previous page.) 
a.

TCGA-KIRC

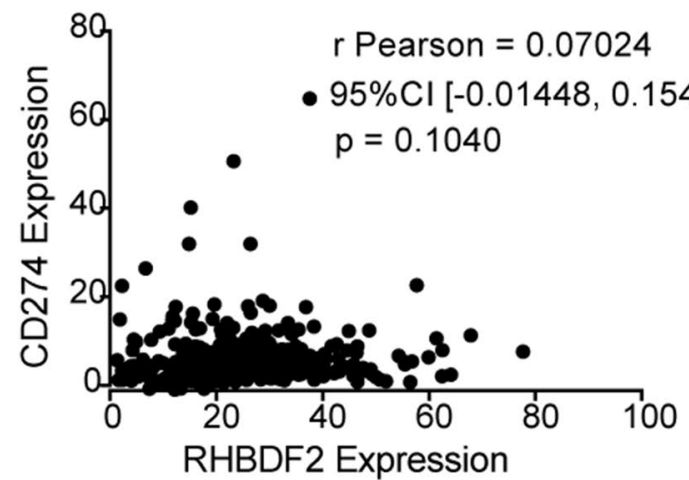

b.

GSE68417

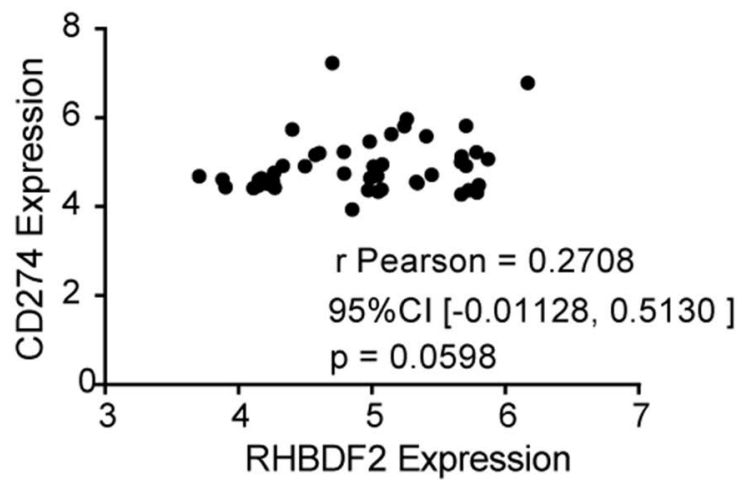

e.

$786-0$

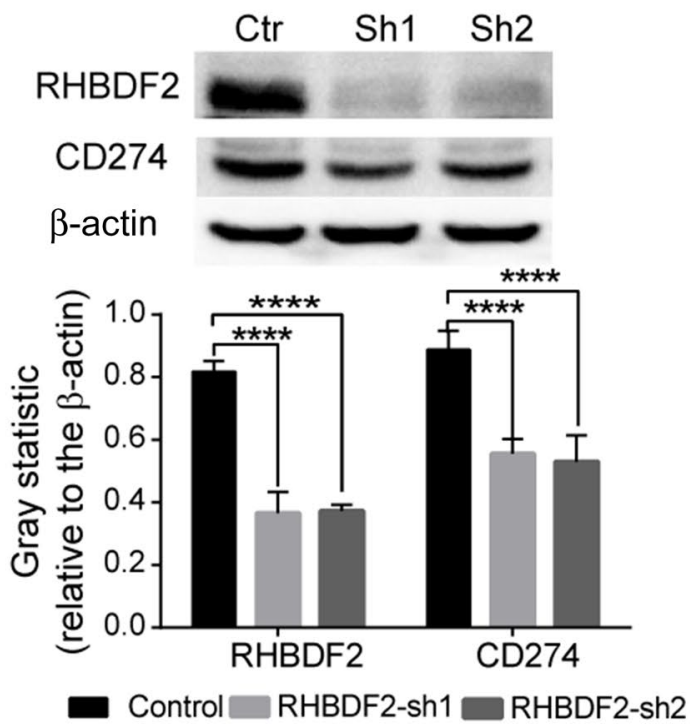

C.

TCGA-KIRC

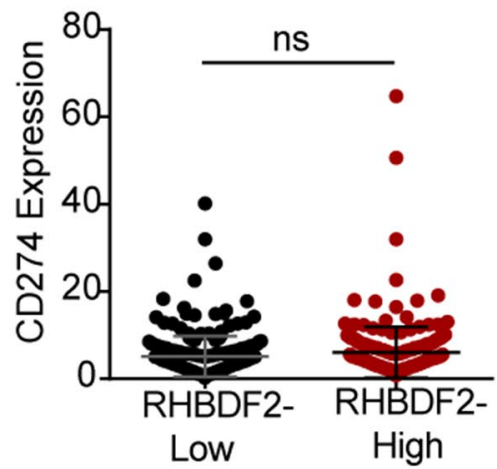

d.

\section{GSE68417}

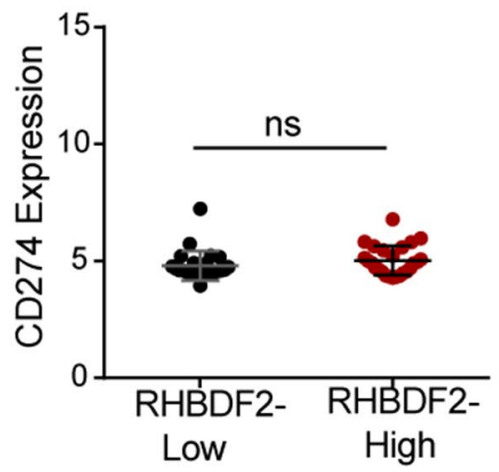

f.

769-P
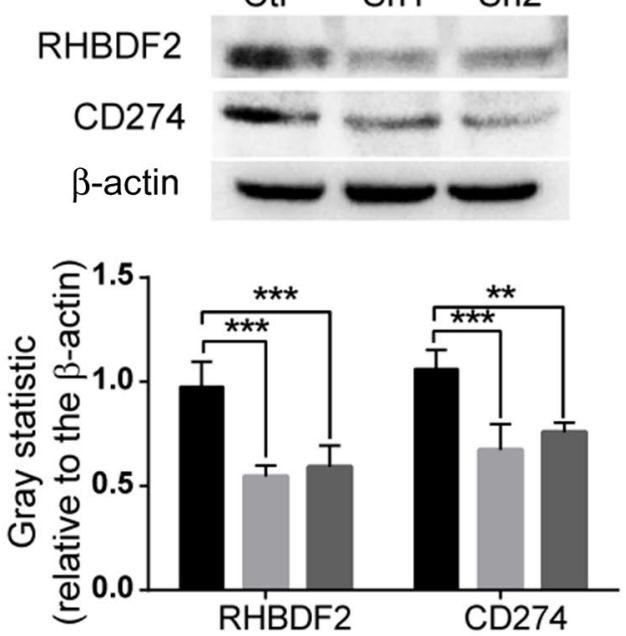

Control RHBDF2-sh1 RHBDF2-sh2

Fig. 6 Immune checkpoints analysis. a Pearson correlation between CD274 and RHBDF2 expression in renal clear cell carcinoma in TCGA database and in b GSE68417 datasets. c RNA sequencing dada of CD274 expressing in renal clear cell carcinoma in TCGA database (RHBDF2-low, $\mathrm{n}=269$; RHBDF2-high, $n=269$; unpaired t-test) and in $\mathbf{d}$ GSE68417 datasets (RHBDF2-low, $n=24$; RHBDF2-high, $n=25$; unpaired t-test). e, $\mathbf{f}$ Protein levels of PD-L1 in RHBDF2-knockdown cell lines, 786-O and 769-P ( $P$-values were from one-way ANOVA, ${ }^{*} p<0.05,{ }^{* *} p<0.01,{ }^{* * *} p<0.005,{ }^{* * *} p<0.001$ ) 


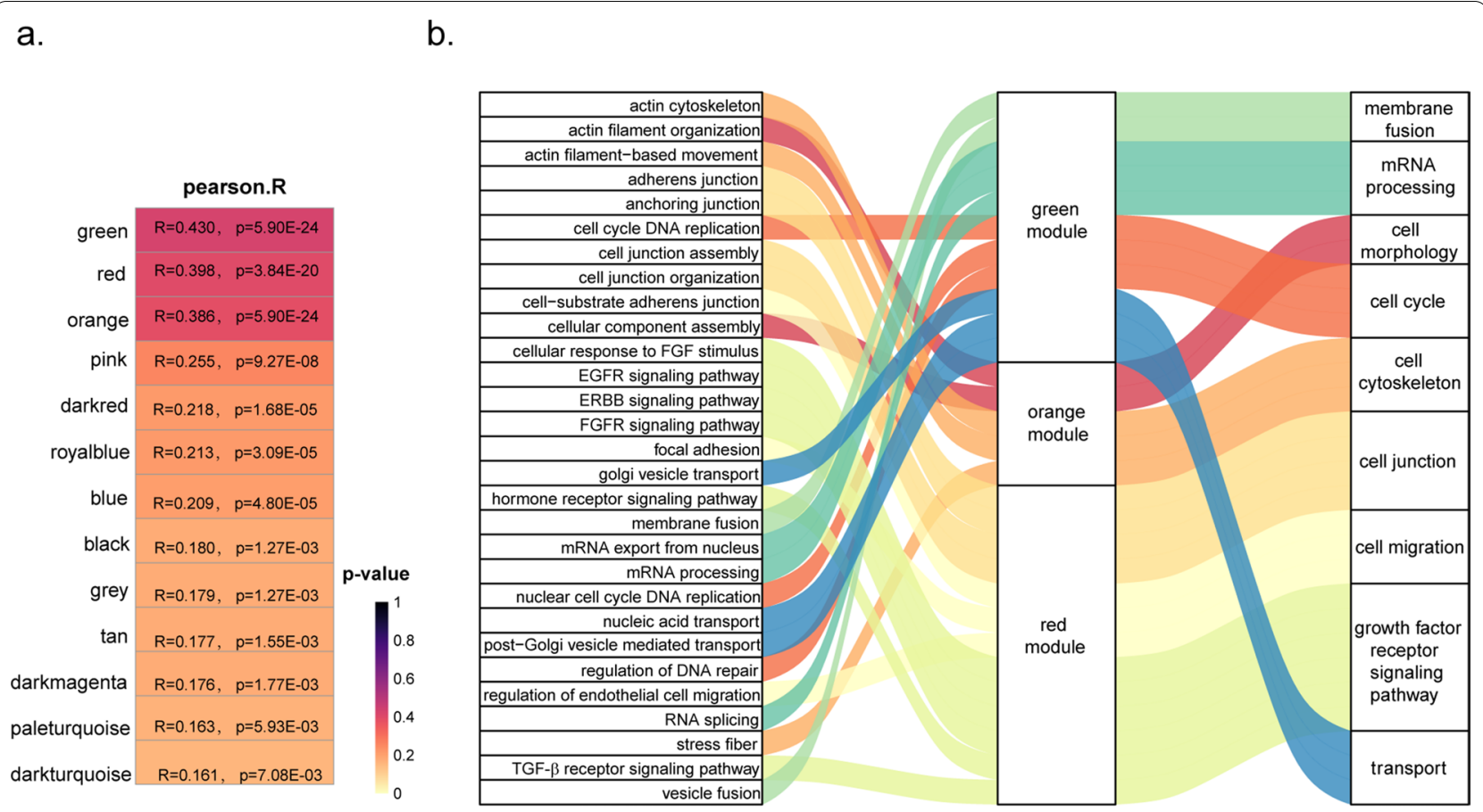

Fig. 7 RHBDF2-related function enrichment. a Relationships between modules and trait analyzed by WGCNA. RHBDF2 expression level was set as a trait, genes in green, red and orange modules had stronger correlation with RHBDF2 expression. b Functional annotation of genes in green, red and orange modules

less clonal and less cytotoxic than those in the immuneactivated group. It is plausible that RHBDF2 over-expression encourages the formation of an immunosuppressive environment. In a recent study [57], we also found that RHBDF1, another member of the proteolytically inactive rhomboid, affected tumor immune microenvironment, through a promotion of endothelial-mesenchymal transition and tumor fibrotic stroma growth. Targeting RHBDF2 either at gene expression or protein function could lead to a release of immunosuppression in tumors and thereby a possible enhancement of the cytotoxicity of immune cells such as macrophages and T-cells.

In future studies, mechanisms underlying the role of RHBDF2 in the modulation of renal clear cell tumor progression and potential impact on the microenvironment are of particular interest. Additionally, in-depth understanding of the plausible linkage between RHBDF2 and PD-L1 is worthy exploration. Such studies may include evaluating the responses to PD-L1 treatment in patients with various levels of RHBDF2. Moreover, RHBDF2 as a relatively large protein molecule and the possibility that it may interact with a number of proteins with critical functions make it an excellent target for therapy development.

\section{Conclusions}

In summary, we found that RHBDF2 is positively correlated with the severity of the malignancy of renal clear cell carcinoma. High expression of RHBDF2 in KIRC is associated with an activation of a number of genes involved in tumor growth and metastasis. Silencing the RHBDF2 gene in renal cancer cells leads to down-regulation of the immunosuppressive checkpoint protein $\mathrm{PD}-\mathrm{L} 1$, even though there is an increase of lymphocyte infiltration into the tumors with high levels of RHBDF2. These findings are consistent with the view that RHBDF2 not only has potential diagnostic and prognostic values as biomarker, it may also be of important value as a therapeutic target in assisting immunotherapy. 
a.

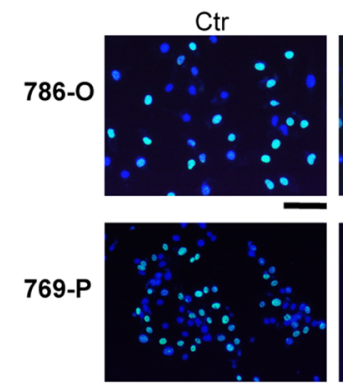

b.

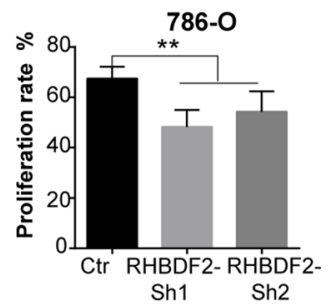

C.
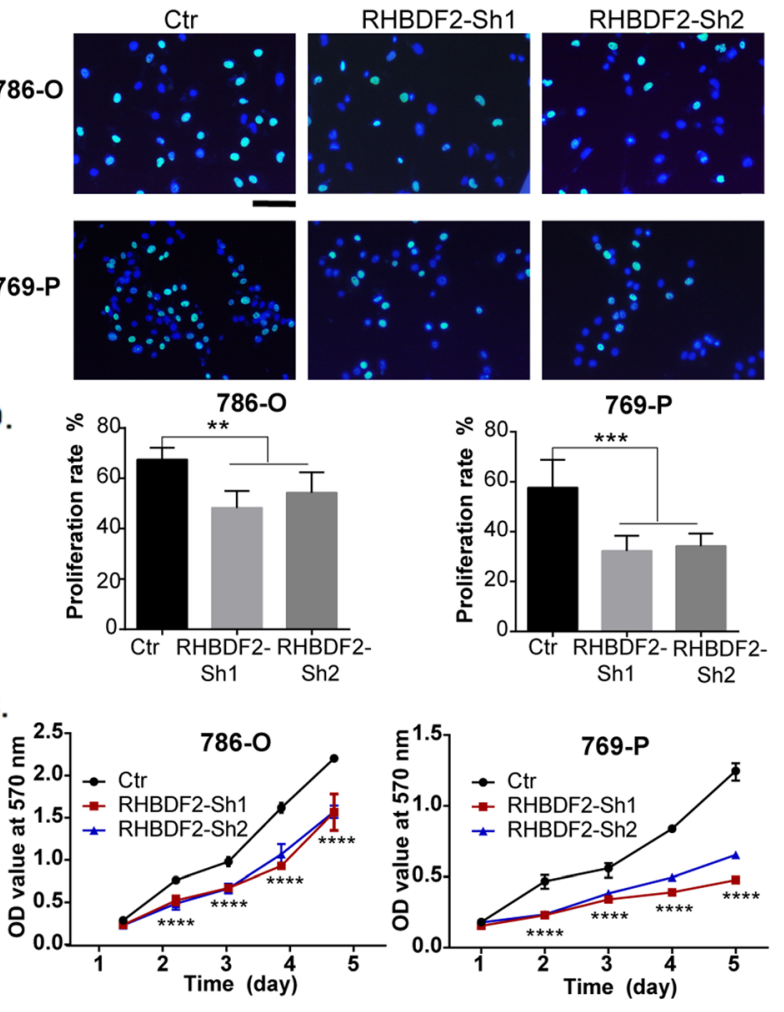

769-P

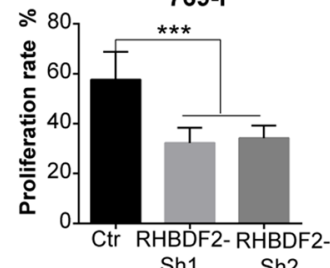

e.

f.

g.

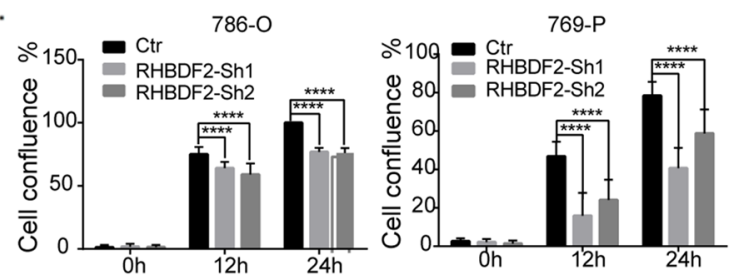

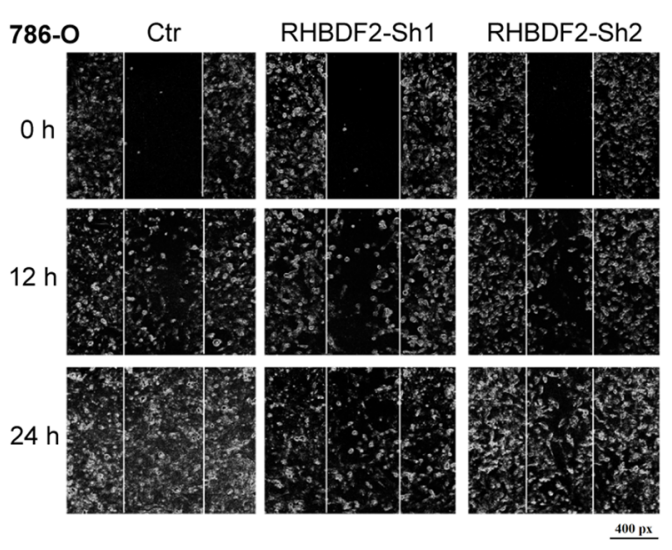

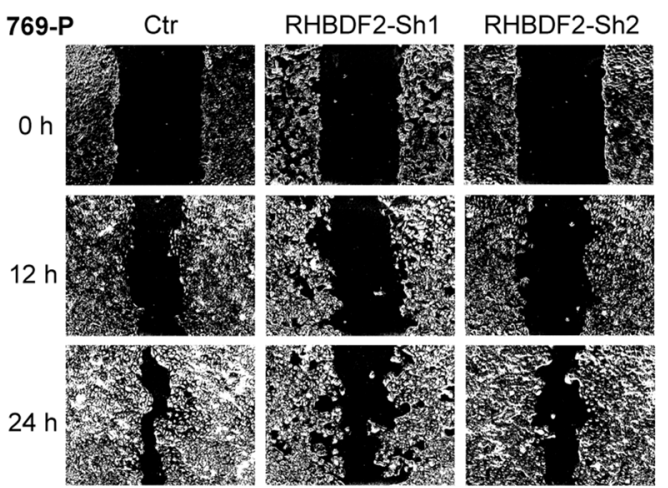

e.

(1) h.

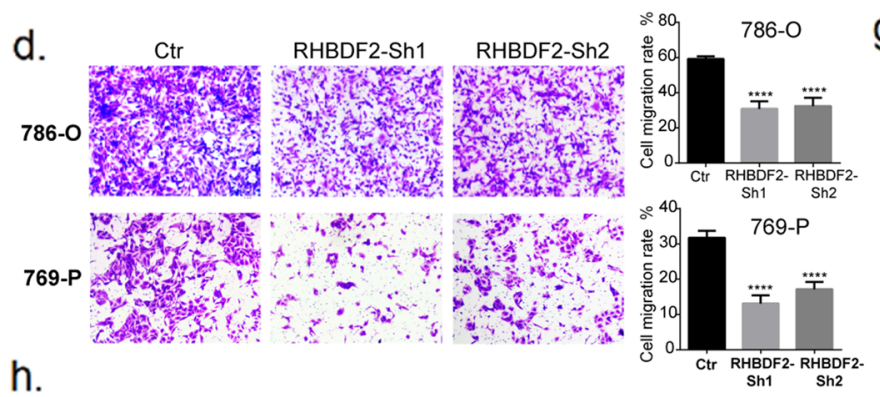

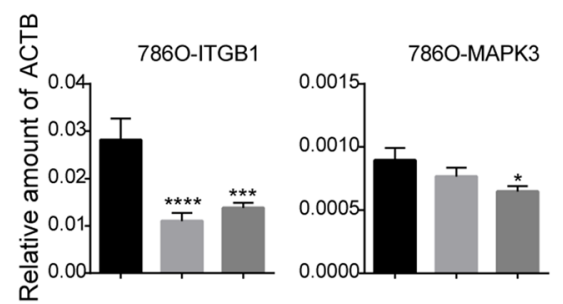
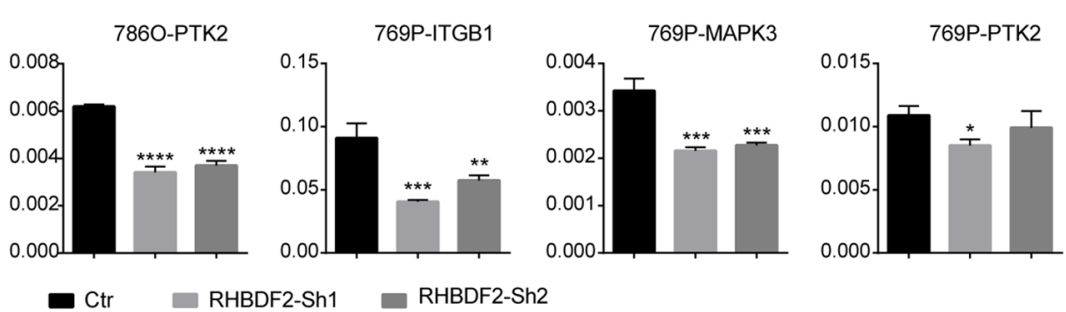

Fig. 8 RHBDF2 knockdown restricted renal clear cell tumor proliferation and migration. a Cell proliferation detection by Edu incorporation assay in 786-O cells and 769-P cells with or without RHBDF2 knockdown. (Blue: nuclei; green: nuclei of S-phase cells.) b Statistics of EDU incorporation rate (t-test). c Statistics of Cell growth speed (one-way ANOVA was used for significance test). $\mathbf{d}$ Cells with or without RHBDF2 knockout were used to evaluate migration ability by Transwell assay. The statistical results were shown on the right side (one-way ANONA). e Cell healing results of 786-O cells and $\mathbf{f} 769-\mathrm{P}$ cells, with or without RHBDF2 knockdown at 0, 12, $24 \mathrm{~h}$ after scratching. $\mathbf{g}$ Statistics of scratching healing (two-way ANOVA). $\mathbf{h}$ mRNA expression of hub genes in 786-O cells and 769-P cells with or without RHBDF2 knockdown (one-way ANOVA). All the results were repeated three times, ${ }^{*} p<0.05,{ }^{* *} p<0.01,{ }^{* * *} p<0.005,{ }^{* * *} p<0.001$ 


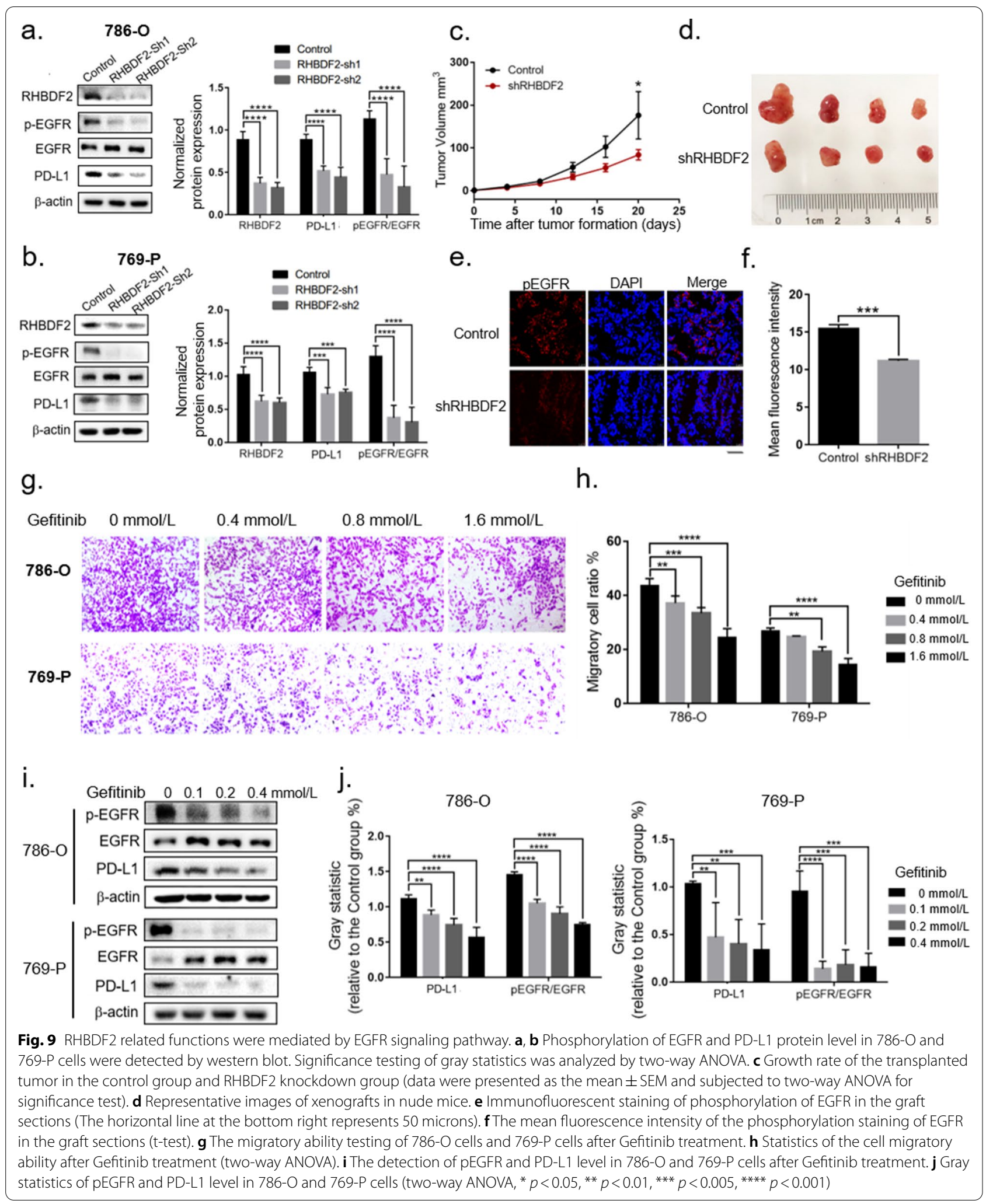




\begin{abstract}
Abbreviations
ACC: Adrenocortical carcinoma; BLCA: Bladder urothelial carcinoma; BRCA : Breast invasive carcinoma; CESC: Cervical squamous cell carcinoma and endocervical adenocarcinoma; CHOL: Cholangiocarcinoma; COAD: Colon adenocarcinoma; DLBC: Diffuse large b-cell lymphoma; DSS: Disease-specific survival; EGFR: Epidermal growth factor receptor; ESCA: Esophageal carcinoma; GBM: Glioblastoma multiforme; GEO: Gene Expression Omnibus; GEPIA Gene Expression Profiling Interactive Analysis; GSEA: Gene Set Enrichment Analysis; GTEx: Genotype-Tissue Expression Project; HNSC: Head and neck squamous cell carcinoma; HR: Hazard Ratio; ICl: Immune checkpoint inhibitors; IMS: Immune infiltration score; KICH: Kidney chromophobe; KIRC: Kidney renal clear cell carcinoma; KIRP: Kidney renal papillary cell carcinoma; LAML: Acute myeloid leukemia; LGG: Brain lower grade glioma; LIHC: Liver hepatocellular carcinoma; LUAD: Lung adenocarcinoma; LUSC: Lung squamous cell carcinoma; MESO: Mesothelioma; OS: Overall survival; OV: Ovarian serous cystadenocarcinoma; PCPG: Pheochromocytoma and paraganglioma; PFS: Progression-free survival; PRAD: Prostate adenocarcinoma; READ: Rectum adenocarcinoma; SARC: Rectum adenocarcinoma sarcoma; SKCM: Skin cutaneous melanoma; STAD: Stomach adenocarcinoma; TCGA: The Cancer Genome Atlas; TGCT: Testicular germ cell tumor; THCA: Thyroid carcinoma; UCEC: Uterine corpus endometrial carcinoma; UCS: Uterine carcinosarcoma; WGCNA: Weighted Gene Correlation Network Analysis.
\end{abstract}

\section{Supplementary Information}

The online version contains supplementary material available at https://doi. org/10.1186/s12935-021-02277-0.

Additional file 1: Table S1. Univariate Cox regression analysis of rhomboid family genes in cancers from TCGA database.

Additional file 2: Fig. S1. Expression pattern of rhomboid genes in ACC, KIRC, LGG and their adjacent normal tissues. Expression of rhomboid genes in tumor and corresponding normal tissues in ACC, KIRC and LGG were analyzed by GEPIA with data in TCGA and GTEx databases (ANOVA, $\left.{ }^{*} p<0.05\right)$.

Additional file 3: Fig. S2. Expression of immune checkpoint molecules between tumor tissues and normal tissues in GEO and KIRC-TCGA datasets. The array of GSE167093 was processed using vst transformation and quantile normalization. The array of GSE68417 was processed with quantile normalization and log2-transformation. The data of GSE126964 was normalized to FPKM value and processed with log2-transformation. The data of KIEC-TCGA was normalized to TPM value and processed with log2-transformation. $P$-values were form one-way ANOVA, ${ }^{*} p<0.05$, ** $p<0.01,{ }^{* * *} p<0.005$, ${ }^{* * *} p<0.001$

Additional file 4: Fig. S3. Immune checkpoints analysis. (a) Pearson correlation of the expression of immune checkpoints and RHBDF2. ( ${ }^{*} p<0.001$, - $p<0.01$ ). (b) Detection of the CD273, CD276 and LGALS9 expression in 786-O and 769-P with or without RHBDF2 knockdown (one-way ANOVA, * $p<0.05,{ }^{* *} p<0.01,{ }^{* * *} p<0.005$, $\left.{ }^{* * *} p<0.001\right)$.

Additional file 5: Fig. S4. Weighted Gene Correlation Network Analysis. (a) Co-expression modules for the KIRC transcriptome. (b) Module genes connectivity and genes significance in green, red and orange modules were present respectively. (c) Module genes significance for RHBDF2 expression and their module membership in green, red and orange modules were present.

Additional file 6: Table S2. Correlation between RHBDF2 level and different modules in WGCNA.

Additional file 7: Table S3. Hub genes of red, green and orange modules in WGCNA.

Additional file 8: Fig. S5. Gene interactive network of hub genes in red module.

Additional file 9: Fig. S6. Gene interactive network of hub genes in green module.

Additional file 10: Fig. S7. Gene interactive network of hub genes in orange module.
Additional file 11: Fig. S8. Functional enrichment analyses of hub genes in red, green and orange modules.

Additional file 12: Table S4. Significant enrichment pathways and functions both in WGCNA and GSEA.

Additional file 13: Fig. S9. Numbers of significant enrichment pathways and functions both in WGCNA and GSEA.

\section{Acknowledgements}

We thank Nankai University for providing experimental platform, and SangerBox, a free online platform for data analysis (http://sangerbox.com/).

\section{Authors' contributions}

ZSZ and LYL designed the study, LW mainly acquired and analyzed data in the research, XXL and YW helped completing cell related experiments. YMY, YYS and SG revised the writing. All authors contributed to the article and approved the submitted version. All authors read and approved the final manuscript.

\section{Funding}

We are grateful for the funding provided by grants from National Natural Science Foundation of China (NSFC) projects (Grant nos. 81972687, 82073064 and 81874167).

\section{Availability of data and materials}

Data in this article were downloaded from publicly available datasets. The datasets for this study can be found in the TCGA database (https://cancergeno me.nih.gov/) and GEO database (https://www.ncbi.nlm.nih.gov/gds).

\section{Declarations}

Ethics approval and consent to participate

This study was ratified by the Ethics Committee of Shanghai Outdo Biotechnology Co., Ltd. And this study does not involve any animal experiments.

\section{Consent for publication}

Not applicable.

\section{Competing interests}

The authors declare that they have no competing interests.

Received: 9 May 2021 Accepted: 18 October 2021

Published online: 04 November 2021

\section{References}

1. Lohi O, Urban S, Freeman M. Diverse substrate recognition mechanisms for rhomboids; thrombomodulin is cleaved by Mammalian rhomboids. Curr Biol. 2004;14(3):236-41.

2. Adrain C, Zettl M, Christova Y, Taylor N, Freeman M. Tumor necrosis factor signaling requires iRhom 2 to promote trafficking and activation of TACE. Science. 2012;335(6065):225-8.

3. Liao HJ, Carpenter G. Regulated intramembrane cleavage of the EGF receptor. Traffic. 2012;13(8):1106-12.

4. Pascall JC, Brown KD. Intramembrane cleavage of ephrinB3 by the human rhomboid family protease, RHBDL2. Biochem Biophys Res Commun. 2004;317(1):244-52

5. Hosur V, Johnson KR, Burzenski LM, Stearns TM, Maser RS, Shultz LD. Rhbdf2 mutations increase its protein stability and drive EGFR hyperactivation through enhanced secretion of amphiregulin. Proc Natl Acad Sci U S A. 2014;111(21):E2200-9.

6. Fleig L, Bergbold N, Sahasrabudhe P, Geiger B, Kaltak L, Lemberg M. Ubiquitin-dependent intramembrane rhomboid protease promotes ERAD of membrane proteins. Mol Cell. 2012;47(4):558-69.

7. Lee WJ, Kim YD, Park J, Shim SM, Lee J, Hong SH, et al. iRhom 1 regulates proteasome activity via PAC1/2 under ER stress. Sci Rep. 2015;5(1):11559.

8. Lacunza E, Rabassa M, Canzoneri R, Pellon-Maison M, Croce M, Aldaz $\mathrm{C}$, et al. Identification of signaling pathways modulated by RHBDD2 in 
breast cancer cells: a link to the unfolded protein response. Cell Stress Chaperones. 2014;19(3):379-88.

9. Canzoneri R, Rabassa M, Gurruchaga A, Ferretti V, Palma S, Isla-Larrain M, et al. Alternative splicing variant of RHBDD2 is associated with cell stress response and breast cancer progression. Oncol Rep. 2018;40(2):909-15.

10. Peng XM, Gao S, Deng HT, Cai HX, Zhou Z, Xiang R, et al. Perturbation of epithelial apicobasal polarity by rhomboid family-1 gene overexpression. FASEB J. 2018;32(10):5577-86.

11. Li J, Bai TR, Gao S, Zhou Z, Peng XM, Zhang LS, et al. Human rhomboid family-1 modulates clathrin coated vesicle-dependent pro-transforming growth factor a membrane trafficking to promote breast cancer progression. EBioMedicine. 2018;36:229-40.

12. Cipolat S, Rudka T, Hartmann D, Costa V, Serneels L, Craessaerts K, et al. Mitochondrial rhomboid PARL regulates cytochrome $c$ release during apoptosis via OPA1-dependent cristae remodeling. Cell. 2006;126(1):163-75.

13. Yan Z, Zou H, Tian F, Grandis JR, Mixson AJ, Lu PY, et al. Human rhomboid family-1 gene silencing causes apoptosis or autophagy to epithelial cancer cells and inhibits xenograft tumor growth. Mol Cancer Ther. 2008;7(6):1355-64.

14. Zou H, Thomas SM, Yan ZW, Grandis JR, Vogt A, Li LY. Human rhomboid family-1 gene RHBDF1 participates in GPCR-mediated transactivation of EGFR growth signals in head and neck squamous cancer cells. FASEB J. 2009;23(2):425-32.

15. Zhou Z, Liu F, Zhang ZS, Shu F, Li LY. Human rhomboid family-1 (RHBDF1) suppresses oxygen-independent degradation of hypoxia-inducible factor-1 alpha in breast cancer. Cancer Res. 2014;74(10):2719-30

16. Song W, Liu W, Zhao H, Li S, Guan X, Ying J, et al. Rhomboid domain containing 1 promotes colorectal cancer growth through activation of the EGFR signalling pathway. Nat Commun. 2015;6:8022.

17. Canzoneri R, Lacunza E, Isla Larrain M, Croce MV, Abba MC. Rhomboid family gene expression profiling in breast normal tissue and tumor samples. Tumour Biol. 2014;35(2):1451-8.

18. Geesala R, Issuree PD, Maretzky T. Novel functions of inactive rhomboid proteins in immunity and disease. J Leukoc Biol. 2019;106(4):823-35.

19. Liu J, Han C, Xie B, Wu Y, Liu S, Chen K, et al. Rhbdd3 controls autoimmunity by suppressing the production of $\mathrm{L}-6$ by dendritic cells via K27-linked ubiquitination of the regulator NEMO. Nat Immunol. 2014;15(7):612-22.

20. Issuree P, Maretzky T, Mcllwain D, Monette S, Qing X, Lang P, et al. iRHOM2 is a critical pathogenic mediator of inflammatory arthritis. J Clin Invest. 2013:123(2):928-32.

21. Bergbold N, Lemberg MK. Emerging role of rhomboid family proteins in mammalian biology and disease. Biochim Biophys Acta. 2013;1828(12):2840-8.

22. Lemberg MK, Freeman M. Functional and evolutionary implications of enhanced genomic analysis of rhomboid intramembrane proteases. Genome Res. 2007;17(11):1634.

23. Adrain C, Strisovsky K, Zettl M, Hu L, Lemberg MK, Freeman M. Mammalian EGF receptor activation by the rhomboid protease RHBDL2. EMBO Rep. 2011;12(5):421-7.

24. Matthew F. The rhomboid-like superfamily: molecular mechanisms and biological roles. Annu Rev Cell Dev Biol. 2014;30(1):235

25. Siegel R, Miller K, Jemal A. Cancer statistics, 2020. CA Cancer J Clin. 2020;70(1):7-30.

26. Hsieh JJ, Purdue MP, Signoretti S, Swanton C, Albiges L, Schmidinger M, et al. Renal cell carcinoma. Nat Rev Dis Primers. 2017:3:17009.

27. Dunnick RN. Renal cell carcinoma: staging and surveillance. Abdominal Radiol. 2016;41(6):1079-85.

28. Petejova N, Martinek A. Renal cell carcinoma: review of etiology, pathophysiology and risk factors. Biomed Pap Med Fac Univ Palacky Olomouc Czech Repub. 2016;160(2):183-94.

29. Zhang H, Zhu G. Predictive biomarkers and novel targets in the treatment of metastatic renal cell carcinoma. Curr Med Chem. 2021;28(25):5213-27.

30. Armas-Alvarez AL, Alois Osorio-Manyari A, Donate-Moreno MJ, VeraBeron R, Salinas-Sánchez AS. Local and distant recurrence of the chromophobe renal cell carcinoma. Arch Esp Urol. 2020;73(1):71-5.

31. Motzer RJ, Escudier B, Mcdermott DF, George S, Hongo F. Nivolumab versus everolimus in advanced renal-cell carcinoma. N Engl I Med. 2015:373(19):917-27.
32. Motzer RJ, Tannir NM, McDermott DF, Frontera OA, Melichar B, Choueiri TK, et al. Nivolumab plus ipilimumab versus sunitinib in advanced renalcell carcinoma. N Engl J Med. 2018;378(14):1227-90.

33. Vuong L, Kotecha RR, Voss MH, Hakimi AA. Tumor microenvironment dynamics in clear-cell renal cell carcinoma. Cancer Discov. 2019;9(10):1349-57.

34. Liu J, Lichtenberg T, Hoadley K, Poisson L, Lazar A, Cherniack A, et al. An integrated TCGA Pan-cancer clinical data resource to drive high-quality survival outcome analytics. Cell. 2018;173(2):400-16.e11.

35. Thibodeau BJ, Fulton M, Fortier LE, Geddes TJ, Pruetz BL, Ahmed S, et al. Characterization of clear cell renal cell carcinoma by gene expression profiling. Urol Oncol. 2016;34(4):168.e1-.e9.

36. Zhao Q, Xue J, Hong B, Qian W, Zhang N. Transcriptomic characterization and innovative molecular classification of clear cell renal cell carcinoma in the Chinese population. Cancer Cell Int. 2020;20(1):461.

37. Alessio V, Margherita G, Orazio P, Massimo C, Chiara D, Elisabetta S, et al. Genome-wide analysis of differentially expressed genes and splicing isoforms in clear cell renal cell carcinoma. PLoS ONE. 2013;8(10):e78452.

38. Laskar RS, Li P, Ecsedi S, Abedi-Ardekani B, Durand G, Robinot N, et al. Sexual dimorphism in cancer: insights from transcriptional signatures in kidney tissue and renal cell carcinoma. Hum Mol Genet. 2021;30(5):343-55.

39. Tang Z, Li C, Kang B, Gao G, Li C, Zhang Z. GEPIA: a web server for cancer and normal gene expression profiling and interactive analyses. Nucleic Acids Res. 2017:45(W1):W98-102.

40. Yoshihara K, Shahmoradgoli M, Martínez E, Vegesna R, Kim H, TorresGarcia W, et al. Inferring tumour purity and stromal and immune cell admixture from expression data. Nat Commun. 2013:4:2612.

41. Li B, Severson E, Pignon J-C, Zhao H, Li T, Novak J, et al. Comprehensive analyses of tumor immunity: implications for cancer immunotherapy. Genome Biol. 2016;17(1):174.

42. Li T, Fan J, Wang B, Traugh N, Chen Q, Liu J, et al. TIMER: a web server for comprehensive analysis of tumor-infiltrating immune cells. Cancer Res. 2017;77(21):e108-10.

43. Li T, Fu J, Zeng Z, Cohen D, Li J, Chen Q, et al. TIMER2.0 for analysis of tumor-infiltrating immune cells. Nucleic Acids Res. 2020;48:W509-14.

44. Zhou G, Othman S, Jessica E, Hancock R, Niladri B, Xia J. NetworkAnalyst 3.0: a visual analytics platform for comprehensive gene expression profiling and meta-analysis. Nucleic Acids Res. 2019;47(W1):W234-41.

45. Xia J, Gill EE, Hancock R. NetworkAnalyst for statistical, visual and network-based meta-analysis of gene expression data. Nat Protoc. 2015;10(6):823-44.

46. Xia J, Benner MJ, Hancock R. NetworkAnalyst—integrative approaches for protein-protein interaction network analysis and visual exploration. Nucleic Acids Res. 2014;42(W1):W167-74.

47. Xia J, Lyle NH, Mayer ML, Pena OM, Hancock RE. INVEX_a web-based tool for integrative visualization of expression data. Bioinformatics. 2013:29(24):3232-4.

48. Xia J, Fjell CD, Mayer ML, Pena OM, Wishart DS, Hancock R. INMEX_a web-based tool for integrative meta-analysis of expression data. Nucleic Acids Res. 2013;41(W1):W63-70.

49. Warde-Farley D, Donaldson SL, Comes O, Zuberi K, Badrawi R, Chao P, et al. The GeneMANIA prediction server: biological network integration for gene prioritization and predicting gene function. Nucleic Acids Res. 2010;38(Web Server issue):W214-20.

50. Rosvall M, Bergstrom CT. Mapping change in large networks. PLoS ONE. 2010;5(1):e8694.

51. Subramanian A, Tamayo P, Mootha VK, Mukherjee S, Ebert BL, Gillette MA, et al. Gene set enrichment analysis: a knowledge-based approach for interpreting genome-wide expression profiles. Proc Natl Acad Sci U S A. 2005;102(43):15545-50

52. Mootha VK, Lindgren CM, Eriksson KF, Subramanian A, Sihag S, Lehar J, et al. PGC-1a-responsive genes involved in oxidative phosphorylation are coordinately downregulated in human diabetes. Nat Genet. 2003;34:264-73.

53. Braun D, Bakouny Z, Hirsch L, Flippot R, Van Allen E, Wu C, et al. Beyond conventional immune-checkpoint inhibition - novel immunotherapies for renal cell carcinoma. Nat Rev Clin Oncol. 2021;18(4):199-214.

54. Xu W, Atkins M, McDermott D. Checkpoint inhibitor immunotherapy in kidney cancer. Nat Rev Urol. 2020;17(3):137-50. 
55. Su L, Guo W, Lou L, Nie S, Zhang Q, Liu Y, et al. EGFR-ERK pathway regulates CSN6 to contribute to PD-L1 expression in glioblastoma. Mol Carcinog. 2020;59(5):520-32.

56. Giraldo NA, Becht E, Vano Y, Petitprez F, Lacroix L, Validire P, et al. Tumorinfiltrating and peripheral blood $T$ cell immunophenotypes predict early relapse in localized clear cell renal cell carcinoma. Clin Cancer Res. 2017;23(15):4416-28.
57. Gao S, Zhang LS, Wang L, Xiao NN, Long H, Yin YL, et al. RHBDF1 promotes AP-1-activated endothelial-mesenchymal transition in tumor fibrotic stroma formation. Signal Transduct Target Ther. 2021;6(1):273.

\section{Publisher's Note}

Springer Nature remains neutral with regard to jurisdictional claims in published maps and institutional affiliations.
Ready to submit your research? Choose BMC and benefit from:

- fast, convenient online submission

- thorough peer review by experienced researchers in your field

- rapid publication on acceptance

- support for research data, including large and complex data types

- gold Open Access which fosters wider collaboration and increased citations

- maximum visibility for your research: over $100 \mathrm{M}$ website views per year

At BMC, research is always in progress.

Learn more biomedcentral.com/submissions 